\title{
EFEITO DOS COMPONENTES DO CAPITAL INTELECTUAL SOBRE O DESEMPENHO FINANCEIRO DE EMPRESAS BRASILEIRAS LISTADAS NO IBRX-50 DA B3 DE 2013 A 2017
}

\author{
EFFECT OF INTELLECTUAL CAPITAL ON THE FINANCIAL \\ PERFORNMANCE OF BRAZILIAN COMPANIES LISTED ON \\ B3's IBRX-50 INDEX FROM 2013 TO 2017
}

\section{EFECTO DEL CAPITAL INTELLECTUAL EN EL DESEMPEÑO FINANCIERO DE LAS EMPRESAS BRASILEÑAS QUE COTIZAN EN EL IBRX-50 DE B3 DE 2013 A 2017}

Chaline Evangelho Meyr

Mestranda em Contabilidade pela Universidade Estadual do Oeste do Paraná, Brasil

chaline.mr@gmail.com

\section{Simone Boruck Klein}

Mestre em Administração pela Fundação Pedro Leopoldo - MG, Brasil

simoneboruck@uol.com.br

\section{Wagner Dantas de Souza Junior}

Docente da Faculdade União das Américas - PR, Brasil

Mestre em Contabilidade pela Universidade Estadual do Oeste do Paraná, Brasil

wagnerdantas9@gmail.com

\section{Denis Dall'Asta}

Docente da Universidade Estadual do Oeste do Paraná, Brasil

Doutor em Engenharia de Produção pela Universidade Federal de Santa Catarina, Brasil denis.asta@unioeste.br

\author{
Contextus \\ ISSNe 2178-9258 \\ Organização: Comitê Científico Interinstitucional \\ Editor-Chefe: Diego de Queiroz Machado \\ Avaliação: double blind review pelo SEER/OJS \\ Recebido em 03/10/2019 \\ Aceito em 02/12/2019 \\ Versão final aceita em 09/12/2019 \\ http://dx.doi.org/10.19094/contextus.v17i3.42289
}

\section{RESUMO}

Este estudo visa analisar o efeito dos componentes do capital intelectual sobre o desempenho financeiro de empresas do índice IBrX-50 da B3, bolsa de valores brasileira. Utilizaram-se os componentes do modelo VAIC'M (PULIC, 1998): o capital humano (HCE), capital estrutural (SCE) e a eficiência do capital empregado (CEE). O desempenho foi medido pelos indicadores financeiros ROA, ROE, ROS e o Valor de Mercado, medido pelo Q de Tobin. Trata-se de uma pesquisa quantitativa, descritiva, documental, com dados secundários de 2013 a 2017 coletados da Economática ${ }^{\circledR}$. Quatro modelos econométricos foram estimados por regressão múltipla com dados em painel para testar as hipóteses. Os resultados indicam que todos os componentes (HCE, SCE e CEE) afetam positivamente o ROA e o ROE; somente o CEE afeta o Valor de Mercado; o HCE afeta positiva, e o SCE negativamente o ROS. Assim, pode-se afirmar que os componentes do capital intelectual afetam alguns dos indicadores de desempenho financeiro em firmas brasileiras. O estudo contribui com a análise empírica dos componentes do capital intelectual no contexto brasileiro. Em benefício das organizações de capital aberto, também contribui para instituir e gerir políticas internas voltadas ao capital intelectual.

Palavras-chave: Capital Intelectual; Valor de Mercado; Desempenho Financeiro; modelo VAICTM; IBrX-50. 


\begin{abstract}
This study aims to analyze the effect of the components of intellectual capital on the financial performance of companies of the B3 IBrX-50 Index, Brazilian stock exchange. The components of the VAIC TM model (PULIC, 1998) were used: human capital (HCE), structural capital (SCE) and employed capital efficiency (EEC). Performance was measured by the financial indicators ROA, ROE, ROS and Market Value, measured by Tobin's Q. This is a quantitative, descriptive, documentary research, with secondary data from 2013 to 2017 collected from Economática ${ }^{\circledR}$. Four econometric models were estimated by multiple regression with panel data to test the hypotheses. Results indicate that all components (HCE, SCE and CEE) positively affect ROA and ROE; only CEE affects Market Value; HCE positively affects and SCE negatively affects ROS. Thus, it can be stated that the components of intellectual capital affect some of the financial performance indicators in Brazilian firms. The study contributes to the empirical analysis of the components of intellectual capital in the Brazilian context. For the benefit of publicly traded organizations, it also contributes to instituting and managing internal policies aimed at intellectual capital.
\end{abstract}

Keywords: Intellectual capital; Market value; Financial performance; VAIC TM model; IBrX50.

\title{
RESUMEN
}

Este estudio tiene como objetivo analizar el efecto de los componentes del capital intelectual en el desempeño financiero de las empresas del Índice IBrX-50 de B3, la bolsa de valores brasileña. Se utilizaron los componentes del modelo VAIC TM (PULIC, 1998): capital humano (HCE), capital estructural (SCE) y eficiencia de capital (CEE). El rendimiento se midió mediante los indicadores financieros ROA, ROE, ROS y Valor de mercado, medido por la Q de Tobin. Esta es una investigación documental cuantitativa, descriptiva, con datos secundarios de 2013 a 2017 recopilados de Economática ${ }^{\circledR}$. Se estimaron cuatro modelos econométricos por regresión múltiple con datos de panel para probar las hipótesis. Los resultados indican que todos los componentes (HCE, SCE y CEE) afectan positivamente el ROA y el ROE; solo la CEE afecta el valor de mercado; HCE afecta positivamente y SCE afecta negativamente a ROS. Por lo tanto, se puede afirmar que los componentes del capital intelectual afectan algunos de los indicadores de desempeño financiero en las empresas brasileñas. El estudio contribuye al análisis empírico de los componentes del capital intelectual en el contexto brasileño. En beneficio de las organizaciones que cotizan en bolsa, también contribuye a instituir y gestionar políticas internas dirigidas al capital intelectual. Palabras clave: Capital intelectual; Valor comercial; Desempeño financiero; modelo VAIC TM; IBrX-50.

\section{INTRODUÇÃO}

A era atual, conhecida como sociedade do conhecimento, resultou de transformações nos meios econômico e social que impactaram as relações entre os indivíduos e seus ambientes (COSMUlESE; GROSU; HLACIUC, 2017). North e Kumta (2018) destacam que, neste período, o conhecimento deve ser considerado o principal recurso ao criar e produzir riqueza, bem-estar social e prosperidade à população. Diante disto, as empresas utilizam o conhecimento como fonte de vantagem competitiva no mercado, pois criar e manter o conhecimento gera valor e sobrevivência para as organizações (EDVINSSON; MALONE, 1998).

Hund, Ruta e Liedgens (2009) destacam um rápido crescimento de empresas baseadas em conhecimento na economia mundial. O capital intelectual, gerado pelas capacidades humanas, tem recebido maior ênfase nas organizações. Ativos intangíveis geram impactos sobre as empresas e fornecem mudanças significativas em diversos aspectos organizacionais (LEV, 2003).

Nas últimas décadas, diversos estudos têm analisado a relação entre o capital intelectual e o desempenho econômico e financeiro das empresas, tais como Bontis, Keov e Richardson 
(2000), Firer e Williams (2003), Chen, Cheng e Hwang (2005), Bollen, Vergauwen e Schnieders (2005), Perez e Famá (2006), Martins, Morais e Isidro (2013), Janošević, DŽenopoljac e Bontis (2013), Sumedrea (2013), Guerrini, Romano e Leardini (2014), Turra et al. (2015), Ozkan, Cakan e Kayacan (2017), Carvalho et al. (2017), González, Calzada e Hernández (2017), Smriti e Das (2018) e Brandt, Machaiewski e Geib (2018). Entre estes, o modelo Value Added Intelectual Capital ou VAICTM ganhou destaque, devido às suas características favoráveis para a comparabilidade entre empresas.

O modelo VAICTM utilizado é composto por indicadores relacionados ao capital empregado, capital humano e capital estrutural que avaliam a eficiência da criação de valor dos recursos tangíveis e intangíveis e suas relações com o desempenho da empresa (PULIC, 1998). O desempenho organizacional pode ser dividido em três categorias: operacional, dos negócios e financeiro (BOLLEN; VERGAUWEN; SCHNIEDERS, 2005). Na abordagem do modelo VAICTM a ênfase está no desempenho financeiro.

Esta pesquisa apresenta-se relevante ao buscar compreender as características de empresas brasileiras quanto ao uso de seu capital intelectual e a sua influência no desempenho de modo a auxiliar acionistas, gestores e profissionais do mercado financeiro a avaliar melhor as empresas e entender o funcionamento atual dos negócios brasileiros. Assim, a questão desta pesquisa é: qual foi o efeito dos componentes do capital intelectual sobre o desempenho financeiro das firmas brasileiras listadas no índice IBrX-50 da B3 de 2013 a 2017 ?

O objetivo do estudo é analisar o efeito dos componentes do capital intelectual sobre o desempenho financeiro das empresas do índice IBrX-50 da B3 de 2013 a 2017. Para tal, o estudo é dividido em etapas, tais como construir o indicador VAIC ${ }^{\text {TM }}$ e seus componentes (capital humano - CEE, capital estrutural - HCE e capital relacional - SCE), para avaliar o capital intelectual das empresas, os indicadores ROA, ROE, ROS e Q de Tobin para avaliar o desempenho financeiro, definir a amostra da pesquisa do IBr-X 50 e aplicar a técnica de regressão múltipla com dados em painel para realizar a análise de dados e discussão da pesquisa. A literatura brasileira tem explorado pouco a relação entre os componentes do capital intelectual e o desempenho econômico e financeiro das organizações. Dessa forma essa pesquisa visa contribuir com esta lacuna ao utilizar o modelo VAICTM e contemplar dados recentes.

A estrutura do trabalho é dividida em cinco seções. Após esta introdução, apresenta-se o referencial teórico do estudo sobre conceito de ativos intangíveis, capital intelectual e de seus modelos de mensuração. Em seguida, na terceira seção, é abordado os aspectos metodológicos da pesquisa, com a definição de variáveis, da amostra e dos modelos econométricos utilizados. 
A quarta seção trata da análise e discussão de dados, enquanto a quinta seção apresenta conclusões, limitações e sugestões de pesquisas futuras do estudo.

\section{REFERENCIAL TEÓRICO}

\subsection{Ativos intangíveis e capital intelectual}

Os ativos intangíveis são fonte de valor para a organização, podendo ser gerados pela inovação, por processos organizacionais ou por recursos humanos únicos em uma empresa. Lev (2001) fornece uma definição para os ativos intangíveis como recursos não físicos, geradores de prováveis benefícios econômicos futuros adquirido por meio de troca ou criação no ambiente interno da empresa, com custos identificáveis e com vida útil limitada e controlada pela organização. Duas características abrangem a expressão "intangíveis" e estão relacionadas à perspectiva de análise de sua classificação, a perspectiva contábil e a perspectiva de gestão.

Na perspectiva contábil de ativos intangíveis, leis e normas oferecem as delimitações quanto ao reconhecimento destes ativos nas demonstrações financeiras, porém estas normatizações ainda estão aquém da equidade de informações necessárias entre as partes interessadas, isto gera uma assimetria de informação entre os agentes econômicos (ANDRIESSON, 2005). Diante das mudanças nas composições das empresas, oriundas da sociedade do conhecimento, avanços têm sido buscados por meio de reformas contábeis para mensurar e reconhecer os ativos intangíveis de maneira mais fidedigna (SKINNER, 2008).

No Brasil, um passo em direção a este objetivo ocorreu pela internacionalização da contabilidade propiciada na Lei 11.638/07, que promoveu harmonização das leis nacionais com as da IAS/IFRS, e institui-se um novo grupo de contas, os ativos intangíveis (BRASIL, 2007). A norma IAS 38, apresenta em 130 parágrafos os aspectos referentes a definições, critérios de reconhecimento, mensuração e divulgação de ativos intangíveis (BRANDT, 2016). Entretanto, mesmo diante das tentativas de reformas contábeis que se referem a mensuração e reconhecimento dos ativos intangíveis não existe consenso na academia nem no mercado quanto ao reconhecimento, mensuração e evidenciação destes ativos (CAÑIBANO; GARCIAAYUSO; SANCHEZ, 2000).

A outra perspectiva de ativos intangíveis tem como escopo a gestão dos ativos intangíveis e utiliza-se de conceitos que apresentam para um material intelectual formalizado, 
capturado e aproveitado, passível de ser convertido em valor monetário e gerador de riqueza ou lucro (EDVINSSON; MALONE, 1998; STEWART, 1998; SULLIVAN JR.; SULLIVAN SR., 2000). Desta forma, os termos "capital intelectual" (CI), "capital do conhecimento" se entrelaçam aos conceitos de ativos intangíveis (ANDRIESSON, 2005). Por esta lente, os ativos intangíveis apresentam-se como similares ao capital intelectual, pois utilizam os capitais intelectuais gerenciáveis, e seus modelos de mensuração têm sido desenvolvidos visando alinhar o valuation das organizações com seus demonstrativos financeiros.

Os conceitos, modelos teóricos e ferramentas de mensuração visam elucidar esta vacuidade existente entre as demonstrações financeiras e o valor de mercado das empresas (OLIVEIRA; NASCIMENTO; DALKIR, 2016). Bontis et al. (1999) já afirmavam em seus estudos que a diferença existente entre o valor de mercado e o valor contábil de uma empresa é explicada pelos ativos intangíveis e que este valor oculto se ampliou nas últimas décadas. $\mathrm{O}$ capital intelectual passou a ser um importante componente das organizações, fonte de vantagem competitiva e transformador das formas de produção, de estratégias e de se conduzir pessoas (VAZ et al., 2015).

Os primeiros apontamentos históricos do conceito de CI surgiram das publicações de Likert (1967) e a de Flamholtz (1974). Entretanto, foi na década de 1970 que Sveiby já tratava das dimensões do capital humano e do CI e as competências e conhecimentos dos empregados foram comparados à avaliação da empresa. Dos estudos de Penrose (1956), Rumelt (1984), Wernerfelt (1984) e Hansen e Wernerfelt (1989), emergiram nesta década os pressupostos da teoria da empresa, em que as competências e conhecimentos técnicos são avaliados como elementos firmes e duradouras além de serem possíveis de desenvolvimento (SULLIVAN, 2000; RACY; MOURA JR.; SCARANO, 2005).

O termo CI foi usado pela primeira vez num artigo da Fortune, porém não tinha o significado de "intelecto", mas sim de um ativo de valor monetário (SULLIVAN, 2000). Os estudos que buscavam a interpretação para este ativo evoluíram e em 1988, Sveiby (1988) propõe uma série de propostas para compor um relatório anual de CI. Porém, foi em 1995 que as mensurações dos ativos intangíveis começaram a ser relatadas ativamente por intermédio do modelo conhecido como Skandia Navigator, de Edvinsson e Sullivan (1996), e fixados às demonstrações financeiras (BONTIS et al., 1999).

O conceito de capital intelectual, segundo Guthrie, Ricceri e Dumay (2012, p. 70), pode ser entendido como "uma técnica de gerenciamento, contabilização e relatórios para entender, 
medir e relatar os recursos de conhecimento, como competências de funcionários, relacionamentos com clientes, marcas, relações financeiras e tecnologias de informação e comunicação". Após estas definições, a seguir são apresentados modelos de mensuração do capital intelectual.

\subsection{Modelos de mensuração do capital intelectual}

Nas últimas décadas um arcabouço teórico mais amplo e rigoroso se desenvolveu e foram levantadas dimensões que compõem o CI. Os três elementos mais predominantes na literatura são: capital humano, capital estrutural e capital relacional; estas dimensões apresentam a interdependência existente entre o conhecimento da empresa (MARTÍN DE CASTRO et al., 2011; SUMEDREA, 2013).

Como capital humano compreende-se o conhecimento e habilidades individuais dos funcionários, conhecimento tácito ou explícitos inerentes às pessoas e sua capacidade de gerar mais conhecimento. O capital estrutural apresenta-se a estrutura que fornece apoio ao capital humano, tais como processos, tecnologias e sistemas diretamente relacionados ao desenvolvimento das atividades da empresa e a combinação do conhecimento formal e informal de maneira eficaz e eficiente, que estrutura e desenvolve a organização (MARTÍN DE CASTRO et al., 2011). No capital relacional lista-se as relações da empresa com o ambiente externo, tais como relacionamento com clientes, confiabilidade da marca, fornecedores e parceiros assim como o valor que a organização dá a estas relações (STEWART, 1998; KAPLAN; NORTON, 2004; MARTÍN DE CASTRO et al., 2011).

Sveiby (1998) afirma que todos os elementos do CI têm como fonte de origem as pessoas, ou seja, resultam de ações humanas. Medir os ativos intangíveis relacionados ao CI é uma tarefa complexa (LEV, 2005), pois criar e extrair o valor por meio de medições e relatórios são passos para a implementação completa de um modelo contábil coerente com a era do conhecimento (OLIVEIRA; NASCIMENTO; DALKIR, 2016).

Modelos teóricos foram desenvolvidos a fim de evidenciar e mensurar o CI, estes modelos são divididos em perspectivas financeiras e não financeiras ou quantitativas e qualitativas do CI. Os modelos de mensuração podem ser categorizados, segundo Sveiby (2010), em quatro dimensões, compreendidos por: métodos diretos de capital intelectual (DIC), 
métodos de capitalização de mercado (MCM), métodos de retorno sobre os ativos (ROA) e métodos de pontuação (SC).

Cada modelo busca avaliar os intangíveis nas organizações sob diferentes formas, seja estimando o seu valor intrínseco e de seus componentes, avaliando o valor de mercado da firma e comparando com o seu patrimônio líquido, avaliando o seu impacto pelo retorno obtido pelas operações da firma, ou por meio de scorecards, ou seja, medidas qualitativas que buscam avaliar a contribuição destes ativos sob o resultado financeiro da firma. No Quadro 1, apresenta-se a tipologia de Sveiby (2010) para classificar os modelos de mensuração de o CI e suas características implícitas.

Quadro 1 - Classificação dos modelos de mensuração de CI

\begin{tabular}{|c|l|}
\hline $\begin{array}{c}\text { Modelos de } \\
\text { mensuração de CI }\end{array}$ & \multicolumn{1}{|c|}{ Características } \\
\hline $\begin{array}{c}\text { Métodos diretos de } \\
\text { capital intelectual (DIC) }\end{array}$ & $\begin{array}{l}\text { Estima o valor financeiro dos ativos intangíveis identificando } \\
\text { seus vários componentes. Uma vez identificados esses } \\
\text { componentes, podem-se avaliar os ativos de forma direta, } \\
\text { individual ou como coeficientes. }\end{array}$ \\
\hline $\begin{array}{c}\text { Métodos de } \\
\text { capitalização de } \\
\text { mercado (MCM) }\end{array}$ & $\begin{array}{l}\text { Calcula a diferença entre a valoração de mercado da companhia } \\
\text { e seu patrimônio líquido com o valor de seu capital intelectual ou } \\
\text { ativos intangíveis. }\end{array}$ \\
\hline $\begin{array}{c}\text { Métodos em retorno dos } \\
\text { ativos (ROA) }\end{array}$ & $\begin{array}{l}\text { Os ganhos médios antes de impostos de uma empresa por um } \\
\text { período são divididos pelos ativos tangíveis médios da empresa. } \\
\text { O resultado é o ROA da empresa que é comparado com a média } \\
\text { do setor. A diferença é multiplicada pelos ativos tangíveis médios } \\
\text { da empresa para calcular um ganho anual médio do intangível. } \\
\text { Dividindo os ganhos acima da média pelo custo médio de capital } \\
\text { da empresa ou uma taxa de juros, pode-se obter uma estimativa } \\
\text { do valor de seus ativos intangíveis ou capital intelectual. }\end{array}$ \\
\hline $\begin{array}{c}\text { Métodos de pontuação } \\
\text { (SC) }\end{array}$ & $\begin{array}{l}\text { Os vários componentes dos ativos intangíveis ou capital } \\
\text { intelectual são identificados e indicadores e índices são gerados } \\
\text { e relatados em scorecards ou como gráficos. Espera-se que } \\
\text { nenhuma estimativa seja feita do valor financeiro dos ativos } \\
\text { intangíveis. Um índice composto pode ou não ser produzido. }\end{array}$ \\
\hline
\end{tabular}

Fonte: adaptado de Sveiby (2010, p. 3).

Cada grupo de métodos de mensuração apresentam vantagens distintas. Os que apresentam perspectivas financeiras como os MCM e ROA são úteis em situações de fusões e aquisições e para as avaliações do mercado de ações, entretanto como construídos sobre normas contábeis já construídas a muito tempo apresentam a desvantagem de não traduzirem a realidade em 
termos monetários. Os métodos DIC e SC fornecem as vantagens de criar uma imagem mais abrangente da saúde organizacional sem olhar para os requisitos financeiros além de apresentar dados factuais. Suas desvantagens são que os indicadores são personalizados a cada contexto e organização o que dificulta comparações entre as organizações (SVEIBY, 2010).

Os principais modelos proeminentes na literatura que privilegiam a abordagem financeira e estão relacionados a criação de valor e de intangíveis nas organizações são: o Q de Tobin, Market-to-Book Value (M/B), Invisible Balance Sheet, EVA®, VAIC ${ }^{\mathrm{TM}}$, e Calculated Intangible Value (SVEIBY, 2010).

\subsection{Características do modelo VAICTM}

Os estudos de Pulic (1998, 2000, 2004) mudaram esta perspectiva de abordagem financeira de controle para criação de valor por meio do modelo VAIC ${ }^{\text {TM }}$. Por intermédio deste modelo, a medição deve ser capaz de monitorar a eficiência de recursos para a criação de valor em uma organização, o objetivo deste método é medir a eficiência dos recursos tangíveis e intangíveis uma organização, independente do seu contexto.

Este modelo mede a eficiência de três tipos de insumos das empresas: capital físico e financeiro, capital humano e capital estrutural, o que o distingue de um modelo exclusivo de medição do capital intelectual, já que a dimensão capital relacional não se encontra nestes modelos e a eficiência dos ativos tangíveis também é mensurada (SVANADZE; KOWALEWSKA, 2015). Portanto, o cálculo do VAIC ${ }^{\text {TM }}$ é resultado do somatório da Equação 1.

$$
\mathrm{VAIC}{ }^{\mathrm{TM}}=\mathrm{CEE}+\mathrm{HCE}+\mathrm{SCE}
$$

Em que:

$\mathrm{CEE}=$ Eficiência do Capital Empregado;

HCE = Eficiência do Capital Humano;

$\mathrm{SCE}=$ Eficiência do Capital Estrutural. 
O modelo VAIC'M é usado para calcular a taxa de eficiência de valor acrescentado, com base na utilização de recursos tangíveis e intangíveis da empresa (CHEN; CHENG; HWANG, 2005). O VAIC ${ }^{\mathrm{TM}}$ é o proxy da eficiência de criação de valor do CI e apresenta-se diretamente proporcional à eficiência da empresa (CHEN GOH, 2005).

Para a composição dos índices de CEE, HCE e SCE é necessário obter o valor agregado (VA) que é representado pela fórmula da Equação 2.

$$
\mathrm{VA}=\mathrm{OP}+\mathrm{CE}+\mathrm{D}+\mathrm{A}
$$

Em que: VA = Valor Agregado;

$\mathrm{OP}=$ Lucro Operacional;

$\mathrm{CE}=$ Despesas com Pessoal;

$\mathrm{D}=$ Depreciação;

$\mathrm{A}=$ Amortização.

A principal característica do modelo VAICTM frente a outros modelos é que se pretende calcular a renda econômica, rotulada de valor agregado, de maneira distinta aos padrões utilizados tradicionalmente, para mensurar o capital intangível nas organizações.

Neste modelo, calcula-se o valor agregado de uma empresa utilizando-se do rendimento global menos as despesas (SVANADZE; KOWALEWSKA, 2015). Trata-se as despesas de trabalho como um ativo, não como um custo. As despesas com pessoal são tratadas como um ativo por Pulic (2000) e é chamada de capital humano. A eficiência deste ativo é calculada por contagem de valor acrescentado quando uma unidade gasta com funcionários se cria (PULIC, 2000). Já o capital empregado é igual ao valor contábil dos ativos líquidos da empresa (FIRER; WILLIAMS, 2003; JANOŠEVIĆ; DŽENOPOLJAC; BONTIS, 2013).

Alguns Autores questionam as suposições usadas no VAIC ${ }^{\text {TM }}$ como base para os cálculos, portanto, este modelo está de frente com uma análise crítica severa (ANDRIESSEN, 2004). Entretanto Firer e Williams (2003) afirmam ser uma fonte padronizada e consistente de medida, e que possibilita uma análise comparativa entre empresas e países. Diante do exposto, apresenta-se a composição do cálculo dos avaliadores de eficiência, conforme as Equações 3, 4 e 5.

$$
\begin{aligned}
& \mathrm{CEE}=\mathrm{VA} / \mathrm{CA} \\
& \mathrm{HCE}=\mathrm{VA} / \mathrm{HC}
\end{aligned}
$$




$$
\mathrm{SCE}=\mathrm{SC} / \mathrm{VA}
$$

Em que:

$\mathrm{CEE}=$ Eficiência do capital empregado;

$\mathrm{VA}=$ Valor agregado;

$\mathrm{CA}=$ Capital empregado, medido pelo Ativo Total da empresa;

$\mathrm{HCE}=$ Eficiência do capital humano;

$\mathrm{HC}=$ Total de salários e encargos durante um ano fiscal;

SCE = Eficiência do capital estrutural;

$\mathrm{SC}=\mathrm{A}$ composição de hardware, software, estrutura organizacional, patentes e marcas registradas, representados no ativo intangível da entidade. Pode ser coletado pela diferença entre o VA e o HC.

\subsection{Estudos anteriores e hipóteses de pesquisa}

Estudos anteriores que analisaram a relação entre o capital intelectual e o desempenho econômico e financeiro de empresas, são apresentados a seguir, em ordem cronológica: Bontis et al. (2000), Firer e Williams (2003), Chen et al. (2005), Bollen et al. (2005), Perez e Famá (2006), Martins, Morais e Isidro (2013), Janošević et al. (2013), Sumedrea (2013), Guerrini et al. (2014), Turra et al. (2015), Ozkan et al. (2017), Carvalho et al. (2017), González, Calzada e Hernández (2017), Smriti e Das (2018) e Brandt, Machaiewski e Geib (2018).

Bontis et al. (2000) investigaram 107 indústrias na Malásia e buscaram a relação existente entre os elementos do capital intelectual: o capital humano, estrutural e de clientes. Os resultados apontam que o capital humano tem influência maior sobre a estruturação da empresa e o desenvolvimento do capital estrutural apresenta relação positiva com o desempenho dos negócios.

Firer e Williams (2003) avaliaram a eficiência do capital intelectual, física, humana e estrutural relacionadas às medidas de desempenho. Foram analisadas 75 empresas de capital aberto na África do Sul. Os resultados apontaram que para aquele contexto, o capital físico continua a se destacar como maior propulsor do desempenho.

Chen et al. (2005) estudaram a relação entre eficiência do capital intelectual e o valor, desempenho financeiro e valor de mercado e utilizaram dados de empresas listadas em Taiwan. 
Os resultados apontam que o capital intelectual influi no desempenho financeiro e no valor de mercado, assim como é um indicador de desempenho futuro.

Bollen et al. (2005) investigaram o valor do capital intelectual e da propriedade intelectual sobre o desempenho de empresas. Utilizaram-se de indústrias farmacêuticas alemãs para amostra. Os resultados mostraram que incluir a propriedade intelectual nestes estudos aumenta a validade estatística e sua relevância para o gerenciamento.

Perez e Famá (2006) investigaram o impacto dos ativos intangíveis não contabilizados no desempenho de empresas norte-americanas com ações na NYSE e NASDAQ. Os resultados apresentaram significância para existência de diferença de desempenho entre as empresas intensas em intangíveis e as empresas intensas em tangíveis.

Martins, Morais e Isidro (2013) determinaram o capital intelectual de empresas portuguesas cotadas na Euronext Lisbon, entre o período de 2001 e 2009, por meio do modelo VAIC ${ }^{\text {TM. }}$ Os resultados do estudo apontam que empresas de alta intensidade tecnológica possuem um VAIC maior que empresas de baixa intensidade. Analisando os componentes do VAIC, foi possível notar que a média do capital humano é nas empresas de alta intensidade tecnológica. Os autores não analisaram a relação entre o VAIC e indicadores de rentabilidade ou desempenho da empresa, porém, sugerem a análise dessa relação em estudos futuros.

Janošević et al. (2013) utilizaram uma amostra de 100 empresas sérvias não financeiras. Foi analisada a relação entre os componentes de eficiência do capital intelectual (VAIC ${ }^{\text {TM}}$ ) e o desempenho financeiro. Os resultados demonstram que a eficiência do capital intelectual não afetou o lucro líquido, a receita operacional e o lucro operacional, porém, os três elementos influenciaram o ROE, enquanto o capital humano e o estrutural influenciaram o ROA das empresas.

Sumedrea (2013) analisou a influência da eficiência do capital intelectual (VAICTM) sobre o desempenho econômico de empresas num período de alta turbulência econômica na Romênia. Os resultados sugerem que a rentabilidade recebe influência do capital financeiro, enquanto o desenvolvimento das empresas é influenciado pelo capital estrutural e humano.

Guerrini et al. (2014), utilizando o VAIC ${ }^{\mathrm{TM}}$, verificaram se a eficiência do capital intelectual afeta o desempenho financeiro presente e futuro de 218 empresas italianas. Os resultados apresentaram influência positiva entre desempenho financeiro e a presença de capital intelectual nas companhias. 
Turra et al. (2015) estudaram a influência do capital intelectual, mensurado pelo VAIC ${ }^{\text {TM }}$, sobre o desempenho financeiro em empresas brasileiras e chilenas. O resultado da pesquisa apontou a existência de uma relação canônica entre os três componentes do capital intelectual em empresas brasileiras e chilenas.

Ozkan et al. (2017) investigaram o desempenho financeiro de 44 bancos na Turquia à luz do modelo VAIC ${ }^{\text {TM. }}$ O resultado apresentou maior influência do capital financeiro e estrutural sobre a rentabilidade destas empresas.

Carvalho et al. (2017) analisaram a influência dos componentes do capital intelectual, medidos pelo VAIC ${ }^{\mathrm{TM}}$ sobre o desempenho econômico e financeiro e sobre o valor de mercado das empresas brasileiras listadas na BM\&FBovespa com dados do período entre 2011 a 2015. Os resultados apontam para uma influência positiva da eficiência do capital aplicado sobre o ROE e do capital humano e estrutural sobre o Q de Tobin, que mediu o valor de mercado das empresas.

González, Calzada e Hernández (2017) investigaram os três componentes do VAICTM, o SCE, HCE e o CEE em empresas do setor industrial do México. Os resultados dos autores apontam que o capital humano é a principal fonte de capital intelectual na criação de valor das empresas industriais mexicanas. Os coeficientes da regressão dos estimadores do SCE, HCE e CEE impactaram positivamente o retorno sobre o ativo (ROA), a capitalização de mercado (medido pelo market-to-book) e o valor de mercado (medido pelo Q de Tobin), assim como tamanho da firma. Smriti e Das (2018), que mediram o efeito do capital intelectual (VAICTM) sobre o desempenho financeiro em empresas indianas listadas no COSPI. Como resultado o capital humano teve grande impacto na produtividade e a eficiência estrutural e de capital empregado influenciaram o crescimento das vendas e o valor de mercado da empresa.

Brandt, Machaiewski e Geib (2018) tiveram o objetivo de calcular em seu trabalho o VAIC ${ }^{\text {TM }}$ em empresas do comércio varejista e avaliar se ele apresenta correlação com os indicadores de rentabilidade ROE e o ROA, utilizando dados contábeis de empresas listadas na BM\&FBovespa de 2010 e 2015. Foi constatado que o indicador VAIC TM exerce correlação positiva com o índice de rentabilidade ROE e não apresentou correlação significativa com o ROA. Quanto à regressão, conclui-se que a variação de 1 ponto percentual em conjunto do ROA e ROE acrescentam uma variação de $6,25 \%$ no VAIC ${ }^{\text {TM. }}$

A partir da realização de diferentes estudos empíricos, como os citados anteriormente, é possível constatar evidências de que o capital intelectual guarda uma relação positiva com o 
desempenho empresarial, pois é considerado um fator determinante para se gerar vantagem competitiva no mercado. Desse modo, o capital intelectual explica a diferença entre o valor de mercado e o valor contábil das empresas (EDVINSSON; MALONE, 1998; STEWART, 1998; SVEIBY, 1997; LEV, 2000; PEREZ; FAMÁ, 2006).

Os componentes de capital intelectual, tal como o CEE, HCE e SCE do modelo VAICTM se propõe em medir a habilidade intelectual das empresas e sugerem como a empresa cria valor, seja pelo know-how de seus funcionários, a estrutura da empresa e a gestão de relacionamento em seu contexto mercadológico, sendo uma indicação do valor futuro da empresa e de sua habilidade de gerar resultados financeiros - afetando o seu desempenho financeiro (SULLIVAN, 2000; GONZÁLEZ; CALZADA; HERNANDEZ, 2017).

Com base no exposto, pretende-se medir empiricamente a relação entre os componentes do capital intelectual CEE, SCE, HCE e indicadores de desempenho financeiro ROA, ROE, ROS e Valor de Mercado no contexto das firmas brasileiras de capital aberto, a fim de compreender esta relação entre capital intelectual e desempenho. Desse modo, quatro hipóteses de pesquisa foram estruturadas:

H1. Quanto maior for o nível dos indicadores CEE, HCE e SCE, maior será o valor de mercado das empresas do IBRx-50 brasileiras.

H2. Quanto maior for o nível dos indicadores CEE, HCE e SCE, maior será o ROA das empresas do IBRx-50 brasileiras.

H3. Quanto maior for o nível dos indicadores CEE, HCE e SCE, maior será o ROE das empresas do IBRx-50 brasileiras.

H4. Quanto maior for o nível dos indicadores CEE, HCE e SCE, maior será o ROS das empresas do IBRx-50 brasileiras.

A próxima seção trata dos procedimentos metodológicos da pesquisa.

\section{METODOLOGIA}

\subsection{Procedimentos e métodos da pesquisa}

O delineamento desta pesquisa a caracteriza como quantitativa, descritiva e procedimentos documental. Quantitativa por receber procedimentos estatísticos, descritiva por 
observar fatos, registrá-los e analisá-los sem a interferência do pesquisador e documental por utilizar de dados sem tratamento analítico (RAUPP; BEUREN, 2006).

O universo da pesquisa compõe-se das 50 empresas utilizadas para gerar o Índice Brasil 50 (IBrX-50) na data de 04/02/2019, excluindo-se a duplicação de dados de duas empresas referentes às ações ordinárias e preferenciais. Estas empresas foram selecionadas por serem as mais negociadas na bolsa de valores brasileira, o que sugere que existam mais investidores individuais e institucionais interessados nos papéis destas empresas, além de analistas para cobrirem o seu desempenho ao longo do tempo. Compreender os fatores que influenciam o desempenho destas firmas é preponderante para este público.

Os dados da pesquisa foram coletados na base Economatica ${ }^{\circledR}$ em novembro de 2018 referente aos exercícios de 2013 a 2017. Os dados não encontrados na base Economatica®, necessários para cálculo de cada um dos componentes do VAIC ${ }^{\text {TM }}$ foram obtidos nas demonstrações contábeis das empresas divulgadas no endereço eletrônico da B3. A necessidade de utilização de duas fontes de coleta justifica a escolha das empresas do Índice Brasil 50 (IBrX50) para composição da amostra.

Os critérios para seleção da amostra foi: possuir todas informações necessárias na base de dados para o cálculo das variáveis e indicadores da pesquisa; não possuir valor nas variáveis dependentes; e não possuir valores extremos (outliers), para não enviesar os estimadores do modelo e não possuírem valores negativos em nenhuma das variáveis estimadas. Assim, foram estimados quatro modelos: o modelo 1 contém 37 empresas e 207 observações; o modelo 2, 32 empresas e 191 observações; o modelo 3, 27 empresas e 157 observações; e o modelo 4, 26 empresas e 151 observações.

\subsection{Variáveis da pesquisa e modelos econométricos}

As variáveis dependentes selecionadas para a pesquisa foram o valor de mercado, o retorno sobre o ativo, o retorno sobre o patrimônio líquido e o retorno sobre as vendas. As variáveis independentes relacionam-se ao indicador do VAICTM desdobrado em três componentes, a eficiência do capital humano, a eficiência do capital estrutural e a eficiência do capital aplicado. O Quadro 2 apresenta as variáveis utilizadas neste estudo, sua descrição, fórmula e autores que as utilizaram, para alcançar os objetivos propostos e atender as hipóteses investigadas. 
Quadro 2 - Variáveis da pesquisa

\begin{tabular}{|c|c|c|c|}
\hline Sigla & Descriçãa & Fórmula & Autores \\
\hline \multicolumn{4}{|c|}{ Variáveis Dependentes } \\
\hline VM & $\begin{array}{l}\text { Valor de } \\
\text { Mercado }\end{array}$ & $\begin{array}{c}\text { Q de Tobin = VMA+VCPC- } \\
\text { VCAC+VCE+VCDPLa /Ativo } \\
\text { Contábil }\end{array}$ & Chung e Pruitt (1994). \\
\hline ROA & $\begin{array}{l}\text { Retorno sobre o } \\
\text { Ativo }\end{array}$ & EBIT/Ativo Contábil & \multirow{3}{*}{$\begin{array}{l}\text { Firer eWilliams (2003); } \\
\text { Janošević et al. (2013). }\end{array}$} \\
\hline ROE & $\begin{array}{l}\text { Retorno sobre o } \\
\text { Patrimônio } \\
\text { Líquido }\end{array}$ & $\begin{array}{c}\text { Lucro líquido/ Patrimônio } \\
\text { Líquido }\end{array}$ & \\
\hline ROS & $\begin{array}{l}\text { Retorno sobre as } \\
\text { Vendas }\end{array}$ & Lucro líquido/Receita líquida & \\
\hline \multicolumn{4}{|c|}{ Variáveis Independentes (Componentes do VAIC ${ }^{\mathrm{TM}}$ ) } \\
\hline $\mathrm{HCE}$ & $\begin{array}{c}\text { Eficiência do } \\
\text { Capital Humano }\end{array}$ & Valor Agregado/Capital Humano & \multirow{3}{*}{$\begin{array}{c}\text { Pulic }(1998,2000,2004), \\
\text { Firer e Williams (2003), } \\
\text { Janošević et al. (2013) e } \\
\text { Chen et al. (2014). }\end{array}$} \\
\hline SCE & $\begin{array}{l}\text { Eficiência do } \\
\text { Capital } \\
\text { Estrutural }\end{array}$ & $\begin{array}{c}\text { Capital Estrutural/Valor } \\
\text { Agregado }\end{array}$ & \\
\hline CEE & $\begin{array}{l}\text { Eficiência do } \\
\text { Capital } \\
\text { Empregado }\end{array}$ & Valor Agregado/Capital Aplicado & \\
\hline
\end{tabular}

Notas: ${ }^{\mathrm{a}} \mathrm{VMA}=$ Valor de mercado da firma $; \mathrm{VCPC}=$ Valor contábil do passivo circulante VCAC $=$ Valor contábil do ativo circulante; $\mathrm{VCE}=$ Valor contábil dos estoques; $\mathrm{VCDLP}=$ Valor contábil da dívida de longo prazo. ${ }^{\mathrm{b}}$ EBIT $=$ Lucro antes dos juros e tributos.

Fonte: elaboração própria.

Quatro modelos econométricos foram empregados na pesquisa para responder as hipóteses definidas na revisão da literatura. O modelo utilizado para a análise de dados será a regressão com dados em painel. Com esta técnica será possível avaliar a influência dos componentes do capital intelectual sobre o desempenho das firmas de capital aberto brasileiras.

$\mathrm{Na}$ Tabela 1, é possível visualizar os modelos econométricos e suas respectivas hipóteses utilizados na pesquisa.

Tabela 1 - Modelos econométricos da pesquisa

\begin{tabular}{c|c}
\hline Hipóteses & Modelos Econométricos \\
\hline $\mathrm{H} 1$ & $V M_{i t}=\beta_{0}+\beta_{1} H C E_{i t}+\beta_{2} S C E_{i t}+\beta_{3} C E E_{i t}+\varepsilon_{i t}(6)$ \\
$\mathrm{H} 2$ & $R O A_{i t}=\beta_{0}+\beta_{1} H C E_{i t}+\beta_{2} S C E_{i t}+\beta_{3} C E E_{i t}+\varepsilon_{i t}(7)$ \\
$\mathrm{H} 3$ & $R O E_{i t}=\beta_{0}+\beta_{1} H C E_{i t}+\beta_{2} S C E_{i t}+\beta_{3} C E E_{i t}+\varepsilon_{i t}(8)$
\end{tabular}


$\mathrm{H} 4$

$$
R O S_{i t}=\beta_{0}+\beta_{1} H C E_{i t}+\beta_{2} S C E_{i t}+\beta_{3} C E E_{i t}+\varepsilon_{i t}(9)
$$

Fonte: elaboração própria.

Desta forma, pode-se proceder a análise e discussão dos resultados da pesquisa.

\section{ANÁLISE E DISCUSSÃO DOS RESULTADOS}

O objetivo geral desta pesquisa é analisar o efeito dos componentes do capital intelectual, CEE, HCE, SCEE e CEE sobre o desempenho financeiro das empresas brasileiras do índice IBrX-50 da B3 de 2013 a 2017, medido pelo VM, ROA, ROE e ROS. Na Tabela 2, apresenta-se o resumo descritivo das variáveis da pesquisa.

Tabela 2 - Estatística descritiva das variáveis

\begin{tabular}{|c|c|c|c|c|}
\hline \multicolumn{5}{|c|}{ Resumo descritivo das variáveis dos modelos } \\
\hline \multicolumn{5}{|l|}{ Modelo 1} \\
\hline & VM & HCE & SCE & CEE \\
\hline Mínimo & 0,84 & 1,04 & 0,43 & 0,07 \\
\hline Média & 2,07 & 2,94 & 0,51 & 0,16 \\
\hline Máximo & 6,19 & 12,68 & 0,92 & 0,61 \\
\hline Desvio-Padrão & 0,98 & 2,31 & 0,22 & 0,09 \\
\hline \multicolumn{5}{|l|}{ Modelo 2} \\
\hline & ROA & HCE & SCE & CEE \\
\hline Mínimo & 0,005 & 1,04 & 0,04 & 0,007 \\
\hline Média & 0,08 & 2,78 & 0,51 & 0,16 \\
\hline Máximo & 0,27 & 11,77 & 0,91 & 0,61 \\
\hline Desvio-Padrão & 0,05 & 1,94 & 0,21 & 0,09 \\
\hline \multicolumn{5}{|l|}{ Modelo 3} \\
\hline & ROE & HCE & SCE & CEE \\
\hline Mínimo & 0,001 & 1,112 & 0,11 & 0,007 \\
\hline Média & 0,17 & 3,16 & 0,55 & 0,16 \\
\hline Máximo & 0,8 & 12,6 & 0,92 & 0,61 \\
\hline Desvio-Padrão & 0,14 & 2,44 & 0,2 & 0,1 \\
\hline \multicolumn{5}{|l|}{ Modelo 4} \\
\hline & ROS & HCE & SCE & CEE \\
\hline Mínimo & 0,02 & 1,12 & 0,11 & 0,07 \\
\hline Média & 0,19 & 3,26 & 0,55 & 0,17 \\
\hline Máximo & 1,81 & 12,68 & 0,92 & 0,61 \\
\hline Desvio-Padrão & 0,32 & 2,53 & 0,20 & 0,10 \\
\hline
\end{tabular}

Fonte: elaboração própria.

Após a apresentação dos dados, foram estimados quatro modelos regressão múltipla em painel. Antes de avaliar os resultados em si, foi necessário avaliar os pressupostos dos modelos 
econométricos calculados. A Tabela 3 apresenta os resultados dos modelos de regressão estimados.

No teste de normalidade dos resíduos, medido pelo Jarque Bera, os modelos 1 ao 4 apresentaram os seguintes resultados, 90,45, 7,3, 181,5 e 25,62, todos significantes estatisticamente a um nível de 5\%. No teste de multicolinearidade, avaliado pelo VIF (Variance Inflation Factor), os resultados dos modelos foram, respectivamente: 2,39, 2,65, 2,66 e 2,68, todos com valores abaixo de 10, o que indica ausência de multicolinearidade entre as variáveis.

No teste de autocorrelação residual, medido pelo Durbin-Watson, os resultados do modelo foram: $0,58,0,78,0,43$ e 0,38 . A literatura sugere que o valor do Durbin-Watson deve ser, via de regra, entre 1,5 e 2,5 para indicar a ausência de correlação serial. No modelo estimado, os valores apontam a existência de autocorrelação residual, sendo necessário a correção do modelo. Com isso, foi aplicado o método de Cochrane-Orcutt, que corrige a autocorrelação residual de erros de primeira ordem, ajustando os resíduos do modelo. Os resultados de Durbin-Watson corrigido foram: 1,83, 2,01, 1,88 e 2,14, sanando este problema de especificação do modelo.

Analisando o ajuste do modelo pelo Teste F, obtemos os resultados de: 41,23, 61,51, 19,71 e 158,71 sendo todos estatisticamente significativos a um nível de $1 \%$. Os resultados do $\mathrm{R}^{2}$ e $\mathrm{R}^{2}$ do modelo sugerem que: no modelo $1,37 \%$ das variações de VM são explicados pelas variações dos componentes do capital intelectual; no modelo 2,49\% das variações de ROA são explicados pelos mesmos componentes; no modelo 3, há $24 \%$ das variações das variáveis independentes sob as variações do ROE; enquanto no modelo 4 , há $76 \%$ das variações das variáveis independentes sob o ROS. A partir daqui, é possível analisar os resultados dos coeficientes do modelo.

No Modelo 1, que analisa a relação entre valor de mercado e os componentes do VAICTM, apresentou um resultado positivo e significativo a um nível de $1 \%$ para o coeficiente do CEE (eficiência do capital empregado). Assim, cada variação em 1 unidade de VM gera uma variação de 6,31 ou 6x no CEE da firma.

No Modelo 2, em que a variável dependente é o ROA, foi possível observar que todos os coeficientes das variáveis HCE, SCE e CEE foram positivos e estatisticamente significantes a um nível de $1 \%$. Os resultados da regressão sugerem que, para firmas brasileiras, a variação de 1 unidade de ROA gera a variação de 0,007 unidades de HCE, 0,06 unidades de SCE e 0,31 unidades de CEE. Assim, a variável que mais gera impacto no ROA é a CEE. 
No Modelo 3, em que a variável dependente é o ROE, todas as variáveis independentes apresentaram resultados dos coeficientes positivos e significativos a um nível de até $5 \%$. Os resultados da regressão indicam que a variação de 1 unidade de ROE é impactada por 0,12 unidades de HCE, 0,15 de SCE e 0,45 de CEE. Do mesmo modo que o ROA, o CEE é a variável que afeta o ROE.

No Modelo 4, em que a variável dependente é o ROS, os resultados foram distintos em relação aos modelos anteriores. Apenas as variáveis HCE e SCE apresentaram coeficientes significativos estatisticamente até um nível de 5\%. Enquanto o HCE (capital humano) afeta positivamente o ROS, o SCE (capital estrutural) afeta negativamente. Assim, pode-se interpretar que para cada variação de 1 unidade de ROS, o HCE impacta esta variável em cerca de 0,12 , e o SCE cerca de $-0,2$. Ou seja, quanto maior o retorno sobre as vendas maior o capital humano empregado e menor o capital estrutural.

Tabela 3 - Modelos de regressão múltipla em painel do estudo

\begin{tabular}{|c|c|c|c|c|}
\hline \multirow[b]{2}{*}{ Variável Dependente } & $\begin{array}{c}\text { Modelo } \\
1\end{array}$ & $\begin{array}{c}\text { Modelo } \\
2\end{array}$ & $\begin{array}{c}\text { Modelo } \\
\mathbf{3}\end{array}$ & Modelo 4 \\
\hline & VM & ROA & ROE & ROS \\
\hline HCE & $\begin{array}{c}-0,005 \\
(0,99)\end{array}$ & $\begin{array}{c}\mathbf{0 , 0 0 7} \\
\left(0,00^{*}\right)\end{array}$ & $\begin{array}{c}\mathbf{0 , 1 2} \\
\left(0,05^{* *}\right)\end{array}$ & $\begin{array}{c}\mathbf{0 , 1 2} \\
\left(0,00^{*}\right)\end{array}$ \\
\hline SCE & $\begin{array}{c}0,41 \\
(0,23)\end{array}$ & $\begin{array}{c}\mathbf{0 , 0 6} \\
\left(0,00^{*}\right)\end{array}$ & $\begin{array}{c}\mathbf{0 , 1 5} \\
(0,04 * *)\end{array}$ & $\begin{array}{c}\mathbf{- 0 , 2} \\
\left(0,03^{* *}\right)\end{array}$ \\
\hline CEE & $\begin{array}{c}\mathbf{6 , 3 1} \\
\left(0,00^{*}\right)\end{array}$ & $\begin{array}{c}\mathbf{0 , 3 1} \\
\left(0,00^{*}\right)\end{array}$ & $\begin{array}{c}\mathbf{0 , 4 5} \\
\left(0,00^{*}\right)\end{array}$ & $\begin{array}{c}0,1 \\
(0,46)\end{array}$ \\
\hline$\beta_{0}$ & $\begin{array}{c}\mathbf{0 , 8 5} \\
\left(0,00^{*}\right)\end{array}$ & $\begin{array}{c}\mathbf{- 0 , 0 1} \\
(0,09 * * *)\end{array}$ & $\begin{array}{l}-0,02 \\
(0,55)\end{array}$ & $\begin{array}{c}\mathbf{- 0 , 1 2} \\
(0,10 * * *)\end{array}$ \\
\hline
\end{tabular}

Pressupostos e Especificações dos Modelos de Regressão

\begin{tabular}{|c|c|c|c|c|c|}
\hline 1 & Normalidade dos resíduos - Jarque Bera & $\begin{array}{c}90,45 \\
(2.3 \mathrm{e}- \\
20 *) \\
\end{array}$ & $\begin{array}{c}7,3 \\
(0,02 * *)\end{array}$ & $\begin{array}{c}181,5 \\
(3.9 \mathrm{e}- \\
\left.40^{*}\right) \\
\end{array}$ & $\begin{array}{c}25,62 \\
(2.7 \mathrm{e}- \\
\left.06^{*}\right)\end{array}$ \\
\hline 2 & Ausência de Multicolinearidade - VIF & 2,39 & 2,65 & 2,66 & 2,68 \\
\hline \multirow{2}{*}{3} & $\begin{array}{llll}\text { Autocorrelação } & \text { Residual } & \text { (AR) } & - \\
\text { Durbin-Watson } & & & \\
\end{array}$ & 0,58 & 0,78 & 0,43 & 0,38 \\
\hline & $\begin{array}{l}\text { Correção da AR pelo método de } \\
\text { Cochrane-Orcutt }\end{array}$ & 1,83 & 2,01 & 1,88 & 2,14 \\
\hline \multirow{4}{*}{4} & $\mathrm{~F}$ & $\begin{array}{l}41,23 \\
\left(0,00^{*}\right)\end{array}$ & $\begin{array}{c}61,51 \\
\left(0,00^{*}\right)\end{array}$ & $\begin{array}{c}17,71 \\
\left(0,00^{*}\right)\end{array}$ & $\begin{array}{l}158,71 \\
\left(0,00^{*}\right)\end{array}$ \\
\hline & $\mathrm{R}^{2}$ & 0,37 & 0,49 & 0,25 & 0,76 \\
\hline & $\mathrm{R}^{2}$ ajustado & 0,37 & 0,49 & 0,24 & 0,76 \\
\hline & $\mathrm{N}^{\circ}$ de observações & 206 & 189 & 156 & 150 \\
\hline
\end{tabular}

Fonte: elaboração própria. 
Com base nos resultados do Modelo 1 de Regressão, não foi possível inferir sobre a hipótese 1 da pesquisa: quanto maior for o nível dos indicadores CEE, HCE e SCE, maior será o valor de mercado das empresas do IBRx-50 brasileiras, pois os indicadores HCE e SCE não apresentaram significância estatística.

Este resultado diferencia-se de outros estudos nesta linha. Chen et al. (2005) constataram que os 3 componentes do VAICTM, HCE, SCE e CEE afetam o valor de mercado de empresas taiwanesas. Smith e Das (2018) verificaram que apenas o SCE e o CEE afetam o valor de mercado das empresas indianas. No contexto brasileiro, Carvalho et al. (2007) verificaram que apenas o HCE e SCE influenciaram o valor de mercado das firmas brasileiras da bolsa de valores como um todo, de 2011 a 2015.

Os resultados dessa pesquisa indicam que somente o CEE impactou o VM das firmas brasileiras do IBrX-50 no período de 2013 a 2017. Isto significa que para a amostra de empresas do estudo, a eficiência do capital empregado, obtida pela maximização do valor agregado (soma do lucro operacional, despesas com pessoal, depreciação e amortização da empresa sobre o ativo total) foi determinante para aumentar o valor de mercado das companhias. Assim, a combinação da utilização de ativos físicos com o investimento em pessoas na organização foi determinante para maximizar o valor agregado das empresas no período e ampliar a percepção do seu valor de mercado para os investidores.

Com base nos resultados do Modelo 2 de Regressão, foi possível aceitar a hipótese 2 da pesquisa: quanto maior for o nível dos indicadores CEE, HCE e SCE, maior será o ROA das empresas do IBRx-50 brasileiras. Este resultado converge com os estudos de González, Calzada e Hernández (2017), que encontraram o mesmo tipo de influência para empresas industriais mexicanas.

Janosenik et al. (2013) obtiveram como achados da pesquisa somente o impacto positivo do capital humano (HCE) e estrutural (SCE) sobre o ROA. No Brasil, Carvalho et al. (2017) verificam que somente CEE influenciaram o ROA. Outros estudos encontraram uma relação positiva entre o VAICTM e os indicadores de desempenho financeiro ROA e ROE (MARTINS; MORAIS; ISIDRO, 2013; SUMEDREA; 2013; GUERRINI et al., 2014; BRANDT; MACHAIEWSKI; GEIB, 2018) porém não avaliaram os componentes do capital intelectual individualmente.

Os resultados desta pesquisa sugerem que CEE, seguido do SCE e do HCE, em ordem, exercem maior impacto sobre o ROA das empresas de capital aberto brasileiros. Com base no 
CEE, compreende-se a maximização do valor agregado, incluindo o investimento em pessoal, é responsável por garantir um maior retorno operacional das empresas.

Na ótica do SCE, que corresponde a razão entre o SC (investimento em intangível) e o (VA) valor agregado, pode-se intuir que quanto maior o investimento em intangível sobre o valor agregado da companhia maior tende a ser o nível de SCE e maior o ROA da empresa. Este resultado é importante para justificar os investimentos em ativos intangíveis nas companhias, como marcas, patentes e tecnologia no geral, e compreender o papel que estes ativos exercem no resultado operacional das empresas.

Analisando a relação entre o ROA e o HCE, que corresponde a razão entre o VA (valor agregado) e o HC (total de salários e encargos), verificou-se que quanto maior o HCE maior tende a ser o ROA da empresa.

Para maximizar HCE, a firma não pode reduzir o seu VA, pois consequentemente irá reduzir o seu lucro operacional e reduzir o ROA. Resta então a empresa ampliar o investimento na remuneração em funcionários (salários) em troca de produtividade laboral. Este resultado é importante para compreender como as políticas de remuneração de pessoal e estabelecimento de uma estrutura organizacional que estimule a produtividade são determinantes para o desempenho operacional das empresas brasileiras.

Com base nos resultados do Modelo 3 de Regressão, foi possível aceitar a hipótese 3 da pesquisa: quanto maior for o nível dos indicadores CEE, HCE e SCE, maior será o ROE das empresas do IBRx-50 brasileiras. Estes resultados compactuam com a pesquisa de Janosenik et al. (2013) e diferem de Carvalho et al. (2017) no contexto brasileiro, que encontraram uma relação positiva somente entre o CEE e o ROE em firmas de capital aberto. Os resultados podem ser interpretados da mesma forma que os resultados obtidos em relação ao ROA, na hipótese 2 do estudo.

Um adendo que se faz é que foi possível observar que os resultados dos coeficientes do HCE, SCE e CEE são maiores sobre o ROE do que sobre o ROA, o que indica que o capital intelectual afeta mais o retorno sobre o patrimônio líquido dos acionistas do que sobre a operação em geral. Isto indica que o tema em debate deve ser de interesse amplo aos acionistas das companhias abertas, sejam eles majoritários ou minoritários.

Com base nos resultados do Modelo 4 de Regressão, não foi possível inferir sobre a hipótese 4 da pesquisa: quanto maior for o nível dos indicadores CEE, HCE e SCE, maior será o ROS das empresas do IBRx-50 brasileiras, pois o indicador CEE não apresentou significância 
estatística. No campo internacional, Smriti e Das (2018) constataram a relação positiva e significativa entre o HCE, SCE e CEE e as vendas nas empresas indianas. Os resultados desta pesquisa sugerem um impacto positivo do HCE e negativo do SCE no o retorno sobre as vendas das empresas. Assim, quanto maior a eficiência do capital humano empregado, maior é o retorno sobre as vendas da companhia. Um resultado um tanto contraditório diz respeito a relação negativa entre o SCE e o ROS, que sugere que quanto menor a eficiência do capital estrutural, maior o retorno sobre as vendas. Pode-se entender que quanto menor o investimento em intangível sobre o valor agregado, maior seria o retorno sobre as vendas das companhias brasileiras no período analisado.

No Modelo 4, os resultados indicam que somente o HCE influencia positiva e significativamente o retorno sobre as vendas das firmas. Assim, aceita-se parcialmente a hipótese 4 do estudo, a qual esperava evidenciar que as eficiências do capital humano, estrutural e empregado afetam positivamente o retorno sobre as vendas ao longo do tempo das grandes companhias brasileiras. Janosenik et al. (2013) evidenciaram que nas empresas da Sérvia os componentes do capital intelectual não afetaram lucro líquido, receita operacional e lucro operacional, diferentemente de Smriti e Das (2018) que verificaram, nas empresas indianas, que os capitais estrutural e humano afetam o crescimento das vendas. Desta forma os resultados são discrepantes entre os estudos encontrados. O contexto específico de cada país e empresa pode ser uma justificativa para essa diferença entre os resultados.

Em síntese, os resultados desta pesquisa demonstram que os componentes do capital intelectual afetam total ou parcialmente o desempenho financeiro das firmas brasileiras, analisadas pelos indicador Valor de Mercado, ROA, ROE e ROS. Estes resultados são importantes para compreender o papel do capital intelectual na prática empresarial, e como as organizações devem criar políticas para estimular o seu desenvolvimento interno, a fim de maximizar o seu desempenho financeiro. A diferença obtida neste estudo em relação aos anteriores reside no período analisado (2013 a 2017), a amostra selecionada e o contexto econômico em que as empresas se inserem.

\section{CONCLUSÃO}

Esta pesquisa analisou o efeito dos componentes do capital intelectual sobre o desempenho financeiro das empresas brasileiras do Índice IBrX-50 da B3 de 2013 a 2017. Para 
atender a proposta, utilizou os componentes de eficiência do capital empregado (CEE), do capital humano (HCE) e do capital estrutural (SCE) do modelo VAICTM, e os relacionou com indicadores de desempenho financeiro empresarial ROA, ROE e ROS e Valor de Mercado, medido pelo Q de Tobin.

Por meio de regressão múltipla com dados em painel, foram empregados quatro modelos econométricos para testar quatro hipóteses de pesquisa. Somente as hipóteses 2 e 3 puderam ser confirmadas neste estudo.

Os resultados da pesquisa indicam que quanto maior for o nível dos indicadores CEE, HCE e SCE, maior será o ROA (H2) e o ROE (H3) das empresas do IBRx-50 brasileiras. Estes resultados sugerem que o capital intelectual é um ativo importante nas sociedades de capital aberto, que deve ser gerido para maximizar o desempenho das companhias. Quanto maior for a eficiência do capital empregado, do capital humano e do capital estrutural, melhores serão os indicadores de retorno sobre o ativo (ROA) e retorno sobre o patrimônio líquido (ROE) - com maior impacto sobre o ROE do que em relação ao ROA.

Desse modo, esta pesquisa contribui gerencialmente ao apresentar fatores determinantes do desempenho financeiro empresarial brasileiro, cabendo aos gestores, conselheiros e acionistas da empresa discutirem e implementarem políticas para melhorar a produtividade do capital intelectual nas empresas. Cientificamente, este estudo contribui, junto a um rol de estudos anteriores, ao apresentar evidências empíricas sobre a influência dos componentes do capital intelectual no contexto brasileiro sobre os indicadores de desempenho financeiro. Entre as limitações do limitação do estudo cita-se a amostra pesquisada utilizada, que contemplou somente companhias de capital aberto listadas no índice $\mathrm{IBrX}-50$ da $\mathrm{B} 3$, o período de abrangência selecionado (2013 a 2017) e os indicadores de desempenho financeiro selecionados.

Pesquisas futuras em empresas de capital aberto no contexto brasileiro podem contemplar a utilização de outros indicadores de desempenho, uma amostra maior de firmas ou período de abrangência. Sugere-se também a aplicação de estudos sobre os componentes do VAIC $^{\text {TM }}$ em firmas de capital fechado, cooperativas e empresas de pequeno porte. Seria interessante também realizar um estudo relacionando os componentes do VAIC ${ }^{\text {TM }}$ e o Sistema Dupont de análise financeira. Outra sugestão seria verificar os drivers de valor que influenciam os componentes HCE, SCE e CEE, em um nível mais detalhado de análise contábil e gerencial. 
Dessa forma, novos estudos contribuíram para compreender o efeito que o capital intelectual exerce sobre o desempenho das organizações brasileiras.

\section{REFERÊNCIAS}

ANDRIESSEN, D. IC valuation and measurement: classifying the state of the art. Journal of $\begin{array}{lllllll}\text { Intellectual Capital, } & \text { v. } 5, \quad \text { n. 230-242, 2004, }\end{array}$ <http://dx.doi.org/10.1108/14691930410533669>.

ANDRIESSON, D. Implementing the KPMG Value Explorer: Critical success factors for applying IC measurement tools. Journal of Intellectual Capital, v. 6, n. 4, p. 474-488, 2005, <http://dx.doi.org/10.1108/14691930510628771>.

BOLLEN, L.; VERGAUWEN, P.; SCHNIEDERS, S. Linking intellectual capital and intellectual property to company performance. Management Decision, v. 43, n. 9, p. 11611185, 2005, <http://dx.doi.org/10.1108/00251740510626254>.

BONTIS, N. et al. The knowledge toolbox: A review of the tools available to measure and manage intangible resources. European Management journal, v. 17, n. 4, p. 391-402, 1999, <http://dx.doi.org/10.1016/S0263-2373(99)00019-5>.

BONTIS, N.; KEOW, C. C.; RICHARDSON, S. Intellectual capital and business performance in Malaysian industries. Journal of intellectual capital, v. 1, n. 1, p. 85-100, 2000, <http://dx.doi.org/10.1108/14691930010324188>.

BRANDT, V. A. Contabilidade e relato dos intangíveis: Análise das empresas cotadas na BM\&FBOVESPA. Tese (Doutorado em Contabilidade, Universidade do Minho (reconhecido pela USP), São Paulo, Brasil, 2016.

BRANDT, V. A.; MACHAIEWSKI, S.; GEIB, V. Intellectual capital and its relationship with retailer trade business profitability indexes listed in BM\&FBOVESPA. BASE-Revista de Administração e Contabilidade da Unisinos, v. 15, n. 4, p. 255-263, 2018, <http://dx.doi.org/10.4013/base.2018.154.01>.

BRASIL. Lei $\mathrm{n}^{\circ}$. 11.638, de 28 de dezembro de 2007. Dispõe sobre a obrigatoriedade de publicação das demonstrações financeiras. Diário Oficial da União, Brasília, DF, 28 de dezembro. 2007. Disponível em: <http://www.planalto.gov.br/ccivil_03/_ato20072010/2007/lei/111638.htm>. Acesso em dez. 2019.

CARVALHO, F. M. R.; DARADDA, L. F. R.; DAL VESCO, D. G.; FIIRST, C. A Influência dos Componentes de Capital Intelectual no Desempenho Econômico/Financeiro e Valor de Mercado (Q-Tobin) nas Empresas Brasileiras Listadas na BM\&FBovespa. In: XVII USP INTERNATIONAL CONFERENCE ACCOUNTING. Anais...São Paulo, Brasil, USP, 2017.

CHEN, F.; LIU, Z.; KWEH, Q. L. Intellectual capital and productivity of Malaysian general insurers. Economic Modelling, v. 36, p. 413-420, 2014, <https://doi.org/10.1016/j.econmod.2013.10.008>. 
CHEN, M.; CHENG, S.; HWANG, Y. An empirical investigation of the relationship between intellectual capital and firms' market value and financial performance. Journal of Intellectual Capital, v. 6, n. 2, p. 159-176, 2005.

CHEN GOH, P. Intellectual capital performance of commercial banks in Malaysia. Journal of $\begin{array}{llllllll}\text { Intellectual Capital, } & \text { v. } & 6, & \text { n. } & 3, & \text { p. } & 385-396, & 2005 \text {, }\end{array}$ <https://doi.org/10.1108/14691930510611120>.

CHUNG, K. H.; PRUITT, S. W. A simple approximation of Tobin's q. Financial Management, p. 70-74, 1994, <https://doi.org/10.2307/3665623>.

COSMULESE, C. G. L.; GROSU, V.; HLACIUC, Elena. Intangible assets with a high degree of difficulty in estimating their value. Ecoforum Journal, v. 6, n. 3, 2017.

EDVINSSON, L.; MALONE, M. S. Capital intelectual: descobrindo o valor real de sua empresa pela identificação de seus valores internos. São Paulo: Makron Books, 1998.

EDVINSSON, L.; SULLIVAN, P. Developing a model for managing intellectual capital. European Management Journal, v. 14, n. 4, p. 356-364, 1996, <https://doi.org/10.1016/02632373(96)00022-9>.

FIRER, S.; WILLIAMS, S. M. Intellectual capital and traditional measures of corporate performance. Journal of Intellectual Capital, v. 4, n. 3, p. 348-360, 2003, <https://doi.org/10.1108/14691930310487806>.

GONZÁLEZ, E. V.; CALZADA, M. A. H.; HERNÁNDEZ, B. C. S. La medición del capital intelectual y su impacto en el rendimiento financiero en empresas del sector industrial en México. Contaduría y administración, v. 62, n. 1, p. 184-206, 2017, <http://dx.doi.org/10.1016/j.cya.2016.10.002>.

GUERRINI, A.; ROMANO, G.; LEARDINI, C. Does intellectual capital efficiency affect financial performance? The case of Italian listed firms. International Journal of Learning and Intellectual Capital, v. 11, n. 2, p. 127-148, 2014.

GUTHRIE, J.; RICCERI, F.; DUMAY, J. Reflections and projections: a decade of intellectual capital accounting research. The British Accounting Review, v. 44, n. 2, p. 68-82, 2012, <https://doi.org/10.1016/j.bar.2012.03.004>.

HANSEN, G. S.; WERNERFELT, B. Determinants of firm performance: The relative importance of economic and organizational factors. Strategic Management Journal, v. 10, n. 5, p. 399-411, 1989, <https://doi.org/10.1002/smj.4250100502>.

HUND, A.; RUTA, N.; LIEDGENS, M. Rooting depth and water use efficiency of tropical maize inbred lines, differing in drought tolerance. Plant and Soil, v. 318, n. 1-2, p. 311-325, 2009, <https://doi.org/10.1007/s11104-008-9843-6>.

JANOŠEVIĆ, S.; DŽENOPOLJAC, V.; BONTIS, N. Intellectual capital and financial performance in Serbia. Knowledge and Process Management, v. 20, n. 1, p. 1-11, 2013, <https://doi.org/10.1108/JIC-07-2015-0068>. 
KAPLAN, R. S.; NORTON, D. P. Mapas estratégicos: convertendo ativos intangíveis em resultados tangíveis. Gulf Professional Publishing, 2004.

LEV, B. Intangibles: Management, measurement, and reporting. Brookings Institution Press, 2000 .

LEV, B. Remarks on the measurement, valuation, and reporting of intangible assets. Economic Policy Review, v. 9, n. 3, 2003.

MARTÍN DE CASTRO, G. et al. Towards 'an intellectual capital-based view of the firm': origins and nature. Journal of Business Ethics, v. 98, n. 4, p. 649-662, 2011, <https://doi.org/10.1007/s10551-010-0644-5>.

MARTINS, M.; MORAIS, A.; ISIDRO, H. O valor do Capital Intelectual das empresas portuguesas. Tourism \& Management Studies, p. 848-862, 2013.

NORTH, K.; KUMTA, G. Knowledge management: Value creation through organizational learning. Springer, 2018.

OLIVEIRA, D.; NASCIMENTO, D.; DALKIR, K. The evolution of the intellectual capital concept and measurement. Ciência da Informação, v. 45, n. 3, 2016.

OZKAN, N.; CAKAN, S.; KAYACAN, M. Intellectual capital and financial performance: A study of the Turkish Banking Sector. Borsa Istanbul Review, v. 17, n. 3, p. 190-198, 2017, <https://doi.org/10.1016/j.bir.2016.03.001>.

PENROSE, E. T. Foreign investment and the growth of the firm. The Economic Journal, v. 66, n. 262, p. 220-235, 1956.

PEREZ, M. M.; FAMÁ, R. Ativos intangíveis e o desempenho empresarial. Revista Contabilidade \& Finanças, v. 17, n. 40, p. 7-24, 2006, <https://doi.org/10.1590/S151970772006000100002>.

PULIC, A. Intellectual capital-does it create or destroy value? Measuring Business Excellence, v. 8, n. 1, p. 62-68, 2004, <https://doi.org/10.1108/13683040410524757>.

PULIC, A. Measuring the performance of intellectual potential in knowledge economy. In: 2nd McMaster Word Congress on Measuring and Managing Intellectual Capital by the Austrian Team for Intellectual Potential. Anais... Hamilton, Canadá, Universidade McMaster, 1998.

PULIC, A. VAICTM -an accounting tool for IC management. International Journal of $\begin{array}{lllllll}\text { Technology Management, } & \text { v. } 20, \quad \text { n. } 5-8, & \text { p. } & 702-714, & 2000 \text {, }\end{array}$ <https://doi.org/10.1504/IJTM.2000.002891>.

RACY, J. C.; MOURA JR., Á. A.; SCARANO, P. R. O Conceito de Empresa na Teoria Econômica: Uma Revisão das Principais Contribuições. Revista de Economia Mackenzie, v. 3, n. 3, 2009. 
RAUPP, F. M.; BEUREN, I. M. Metodologia da Pesquisa Aplicável às Ciências. In: BEUREN, I. M. (Org.). Como elaborar trabalhos monográficos em contabilidade: teoria e prática. São Paulo: Atlas, 2006.

RUMELT, R. P. Toward a strategic theory of the firm. In: LAMB, R. B. (Ed.). Competitive Strategic Management. Englewood Cliffs: Prentice-Hall, 1984. p. 556-570.

SKINNER, D. J. Accounting for intangibles-a critical review of policy recommendations. Accounting and Business Research, v. 38, n. 3, p. 191-204, 2008, <https://doi.org/10.1080/00014788.2008.9663332>.

SMRITI, N.; DAS, N. The impact of intellectual capital on firm performance: a study of Indian firms listed in COSPI. Journal of Intellectual Capital, v. 19, n. 5, p. 935-964, 2018, $<$ https://doi.org/10.1108/JIC-11-2017-0156>.

SULLIVAN, P. H. Value driven intellectual capital: how to convert intangible corporate assets into market value. New York: Wiley \& Sons, 2000.

SULLIVAN JR., P. H.; SULLIVAN SR., P. H. Valuing intangibles companies-An intellectual capital approach. Journal of Intellectual Capital, v. 1, n. 4, p. 328-340, 2000.

SUMEDREA, S. Intellectual capital and firm performance: A dynamic relationship in crisis time. Procedia Economics and Finance, v. 6, p. 137-144, 2013, <https://doi.org/10.1016/S2212-5671(13)00125-1>.

STEWART, T. A. Capital intelectual: a nova vantagem competitiva das empresas. Rio de Janeiro: Campus, 1998.

SVANADZE, S.; KOWALEWSKA, M. The measurement of intellectual capital by VAIC method-example of WIG20. Online Journal of Applied Knowledge Management, v. 3, n. 2, p. 36-44, 2015.

SVEIBY, K. E. A nova riqueza das organizações (LET Frazão, Trad.). Rio de Janeiro: Editora Campus, 1998.

SVEIBY, K. E. Methods for Measuring Intangible Assets, 2010. Disponível em: <https://www.sveiby.com/files/pdf/intangiblemethods.pdf>. Acesso em 19 jan. 2019.

TURRA, S. et al. Efeitos do capital intelectual sobre o desempenho financeiro em empresas brasileiras e chilenas. Contextus-Revista Contemporânea de Economia e Gestão, v. 13, n. 2, p. 82-104, 2015, <https://doi.org/10.19094/contextus.v13i2.637>.

VAZ, C. R. et al. Capital intelectual: classificação, formas de mensuração e questionamento sobre usos futuros. Navus: Revista de Gestão e Tecnologia, v. 5, n. 2, p. 73-92, 2015.

WERNERFELT, B. A resource-based view of the firm. Strategic Management Journal, v. 5, n. 2, p. 171-180, 1984, 〈https://doi.org/10.1002/smj.4250050207>. 